\title{
Reliable Computation in Noisy Backgrounds Using Real-Time Neuromorphic Hardware
}

\author{
(Invited Paper) \\ Hsi-Ping Wang ${ }^{* \dagger}$, Elisabetta Chicca ${ }^{\ddagger}$, Giacomo Indiveri ${ }^{\ddagger}$ and Terrence J. Sejnowski* ${ }^{* \dagger}$ \\ ${ }^{*}$ Howard Hughes Medical Institute \\ Computational Neurobiology Laboratory \\ Salk Institute, La Jolla, CA 92037 \\ Email: ping@salk.edu and terry@salk.edu \\ ${ }^{\dagger}$ Division of Biological Sciences \\ University of California San Diego \\ La Jolla, CA 92093 \\ $\ddagger$ Institute of Neuroinformatics \\ University of Zurich and Swiss Federal Institute of Technology \\ Zurich, Switzerland \\ Email: chicca@ini.phys.ethz.ch and giacomo@ini.phys.ethz.ch
}

\begin{abstract}
Spike-time based coding of neural information, in contrast to rate coding, requires that neurons reliably and precisely fire spikes in response to repeated identical inputs, despite a high degree of noise from stochastic synaptic firing and extraneous background inputs. We investigated the degree of reliability and precision achievable in various noisy background conditions using real-time neuromorphic VLSI hardware which models integrate-and-fire spiking neurons and dynamic synapses. To do so, we varied two properties of the inputs to a single neuron, synaptic weight and synchrony magnitude (number of synchronously firing pre-synaptic neurons). Thanks to the realtime response properties of the VLSI system we could carry out extensive exploration of the parameter space, and measure the neurons firing rate and reliability in real-time. Reliability of output spiking was primarily influenced by the amount of synchronicity of synaptic input, rather than the synaptic weight of those synapses. These results highlight possible regimes in which real-time neuromorphic systems might be better able to reliably compute with spikes despite noisy input.
\end{abstract}

\section{INTRODUCTION}

A relevant question in neuroscience concerns whether information is encoded in the rates of neural spikes or in the precise spike times of their relative occurrences. One concern facing the viability of the increasingly popular spike-time coding paradigm is that this scheme requires high degrees of reliability and precision in the timing of spikes in response to a given stimulus. In other words, the neural response should be the same given repeated trials of the same input signal, with different noisy background activity [1]. Biological neurons face significant challenges to maintain spike-time reliability from sources as noisy background synaptic inputs, stochastic synaptic release, ionic channel noise, and complex synaptic dynamics which can influence post-synaptic signal integration.

In addition to these challenges, certain neurons face an additional challenge: they are capable of reliably relaying signals they receive from only a small subset of their input synapses. The canonical example of this is the V1 layer 4 cells, which relays information from the lateral geniculate nucleus (LGN) to the visual cortex with only $10 \%$ of their total afferent synapses originating from the LGN [2], [3]. Moreover, in vivo experiments have shown that they are able to do so extremely reliably, not only within the same cell, but also across cells of the same class in different animals, when stimulated with the same visual input [4]. A fundamental question we address is how the experimentally observed high degree of spike timing reliability is possible given such large sources of unreliability and noise.

One hypothesis to explain this effect posits that thalamocortical synapses are stronger than intracortical synapses [5]. However, another hypothesis springs from studies of rat somatosensory cortical neurons suggesting synchronous release of multiple weak synapses from the thalamus as a major contributing mechanism of reliable firing [6]. Synchronous inputs from the LGN to V1 can result from precise connections between the retina and LGN [7], [8]. This synchrony among thalamocortical synapses increases the magnitude and hence the reliability of the evoked post-synaptic response.

In this paper we show how the reliability of a silicon neuron is primarily driven by the synchronous firing of its inputs rather than by increasing the input synaptic weights.These results highlight the possibility of creating real-time noise tolerant spike-time based neuromorphic systems, despite the inherent mismatch and inhomogeneities typical of subthreshold VLSI circuits.

\section{Methods}

\section{A. Neuromorphic Hardware}

The VLSI device used in this work implements an array of 128 low-power Integrate-and-Fire (I\&F) neurons [9] with 4096 synaptic integrator circuits [10]. The chip has been fabricated using a standard AMS $0.35 \mu \mathrm{m}$ CMOS process, and covers an area of about $10 \mathrm{~mm}^{2}$. Each I\&F neuron receives 

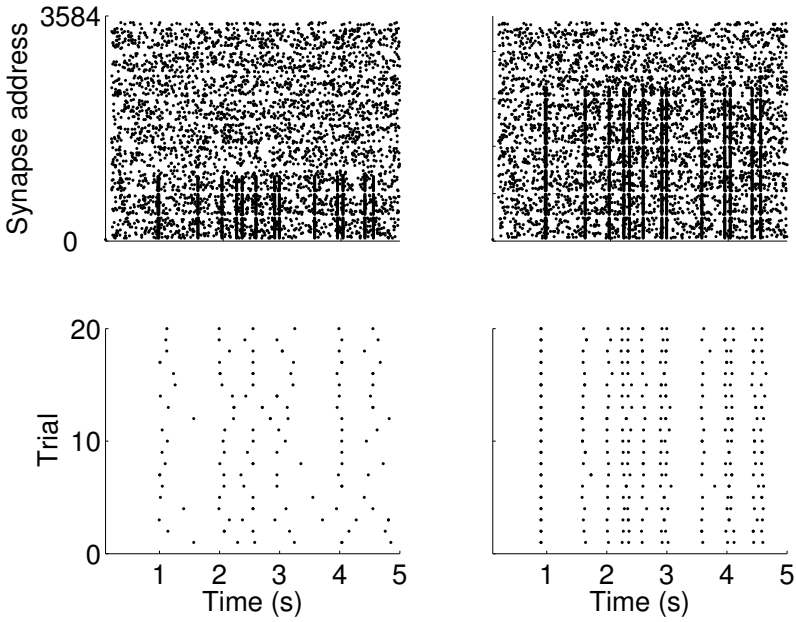

Fig. 1. Input and output raster plots. Top row: example of spike train inputs to the neuron's synapses versus time. The left quadrant shows a trial with synchrony magnitude 1075 (30\% of the synapses receive identical spike trains), and the right quadrant shows a trial with synchrony magnitude of 2508 (70\% of stimulated synapses). Bottom row: output raster plots for 20 different trials, in response to the stimuli with synchrony magnitude 1075 (left quadrant) and 2508 (right quadrant).

input currents from a row of 32 afferent dynamic synapses that receive input spikes via an Address Event Representation (AER) [11] and produce post-synaptic currents (either excitatory or inhibitory depending on their nature) with exponential temporal dynamics. The spiking activity of the neurons is also encoded using the AER. In this representation spikes are real-time asynchronous digital events that carry analog information in their temporal structure. We interfaced the chip to a workstation, via a custom printed circuit board (PCB) with an AER to USB interface. This board allows us to stimulate the synapses on the chip (e.g. with synthetic trains of spikes), and monitor the activity of the I\&F neurons with a maximum bandwidth of $40 \mathrm{MB} / \mathrm{s}$. We carried out prototyping experiments by stimulating the synapses on the chip and monitoring the neuron's response, in real-time. A dedicated on-chip line multiplexer can be used to reconfigure the on-chip network topology: depending on the multiplexer's state, the number of synapses connected to the number of neurons $\left[n_{S}, n_{N}\right]$ can be $[32,128],[64,64],[128,32]$, all the way to $[4096,1]$. In the following experiments, we configured the chip to act as a single neuron connected to all 4096 synapses on the chip.

\section{B. Synapses}

The silicon synapses used in the chip are "diff-pair integrator" circuits [10] with a global tunable time constant parameter $\left(V_{\tau}\right)$, and a global synaptic efficacy parameter $\left(V_{w}\right)$. Of all the 4096 synapses, 3584 have the same global parameters, while the remaining can be configured as inhibitory synapses and short-term depressing synapses.

In our experiments, we simulated the 3584 excitatory synapses with independent random spike trains with Poisson distributed arrival times to emulate background noise. This background was recomputed for each trial. On top of this noisy background, we varied the number of synapses receiving a common Poisson distributed synchronous input. By doing so, we are in some sense emulating thalamocortical synapses receiving common signal events.

The background pre-synaptic firing rate of the intracortical excitatory synapses was set to $3 \mathrm{~Hz}$, based on estimates obtained from awake animals in vivo [12]. The synaptic weights of the background excitatory and inhibitory synapses were also varied systematically.

\section{Calculation of reliability}

Reliability was computed using a synchrony-based measure applied to the spike train output of the model neuron [13]. Briefly, each spike train obtained from the $\mathrm{N}$ repeated presentations of the same stimulus and different background was convolved with a Gaussian filter of width $2 \sigma$. The resulting smoothed trains are denoted with $\vec{s}_{i}$ (for the $i^{t h}$ trial). The sum of the inner product between all pairs of trials (each trial normalized by its norm) is divided by the number of pairs to yield a reliability measure ranging from zero to one. Formally, the reliability $\mathrm{R}$ is defined as

$$
R=\frac{2}{N(N-1)} \sum_{i=1}^{N} \sum_{j=i+1}^{N} \frac{\vec{s}_{i} \cdot \vec{s}_{j}}{\left|\vec{s}_{i}\right| \cdot\left|\vec{s}_{j}\right|}
$$

The $\sigma$ of the Gaussian kernel was set to $3 \mathrm{msec}$ based on the observation that reliable events in the experimental data for V1 cells occur with a jitter of approximately 3 msec [14] and $\mathrm{N}=20$ trials. As this is computed as a single scalar metric across trials and does not have a variance, error bars are not generated. However, greater confidence levels for this metric can be increased with larger $\mathrm{N}$.

\section{Synchrony Magnitude}

The total duration of a trial was 5 seconds. For a set of trials the inputs consisted of a pattern of signal events which were received simultaneously by some designated subset of the synapses embedded in the random $3 \mathrm{~Hz}$ background activity. The number of synapses in the target subset is termed the synchrony magnitude. Figure 1 (top-left quadrant) shows a typical input with synchrony magnitude 1075 (i.e. $30 \%$ simultaneously stimulated synapses). Figure 1 (top-right quadrant) shows a typical input with synchrony magnitude 2508 (i.e. $70 \%$ simultaneously stimulated synapses). Trial to trial randomness was obtained by changing the background activity randomly. The pre-synaptic signal events, however, were identical from trial to trial. The resulting outputs from 20 trials are shown in raster form in the bottom row of Fig. 1. These outputs are then fed to our reliability analysis to generate a single scalar reliability measure.

\section{RESUlTS}

\section{A. Variation of synaptic drive}

Reliability of cortical neurons could depend more on excitatory post-synaptic potentials (EPSPs) from thalamic afferents on a V1 cell than on other intracortical inputs [12], [15]. To 


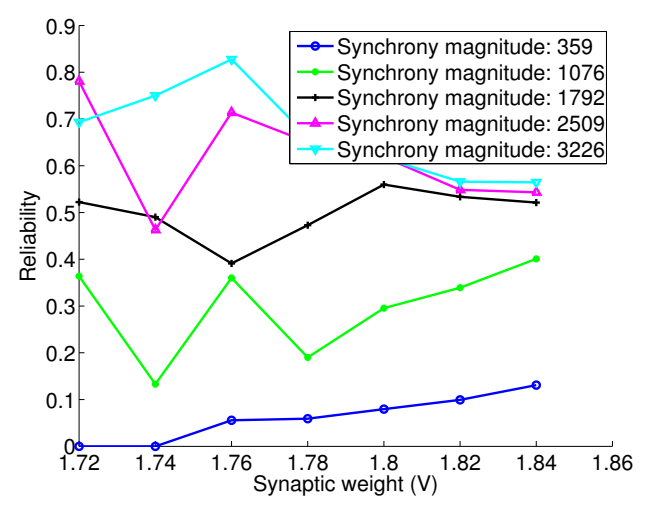

Fig. 2. Reliability versus synaptic weight strength, for increasing values of synchrony magnitude.

test this hypothesis in our hardware setup we systematically increased the EPSP amplitudes, by varying the synaptic circuit's bias voltage $V_{w}$. The range of bias voltages required to produce EPSPs with biologically plausible amplitudes was experimentally found to vary from $1.72 \mathrm{~V}$ to $1.84 \mathrm{~V}$. The current produced by the synaptic circuits depends exponentially on $V_{w}$, and the effect of varying this bias from the minimum to the maximum of this range was equivalent to scaling the synaptic efficacy from an initial nominal value to a final value three times as large as the initial one.

Figure 2 shows how the reliability measure changes with synaptic strength, for different values of synaptic magnitude. As shown, there is little dependence of the reliability to the synaptic weight bias voltage, independent of the synchrony magnitude.

\section{B. Effects of synchrony magnitude on reliability}

To study the effects of synchronous synaptic inputs on the reliability of repeated presentations of the same stimulus, we stimulated a group of synapses, representing thalamocortical input synapses, with synchronous input spikes. These synchronous inputs represent the signal event transmitted from the LGN in response to a visual stimulus presented in the neuron's receptive field.

The number of stimulated synapses was varied in each experiment with the synchrony magnitude, as described in Sec. II-D. Figure 4 shows that the output firing rate increases as synchrony magnitude is increased. This holds true for all synaptic weights. However each experiment only increased by a few hertz on average from the increase in synchrony. By far the greater influence on firing rate was from differing synaptic weights (i.e. the spread between different lines was greater than the rise of each individual line itself). Figure 3 shows that the reliability consistently rose, independent of the synaptic weight, for each experiment.

These results indicate that while the neuron's mean firing rate depends strongly on the values of the synaptic weights, its reliability is determined mainly by the synchrony magnitude.

The observation that firing rate had little relation to the spike time reliability in these experiments further confirm that a non-

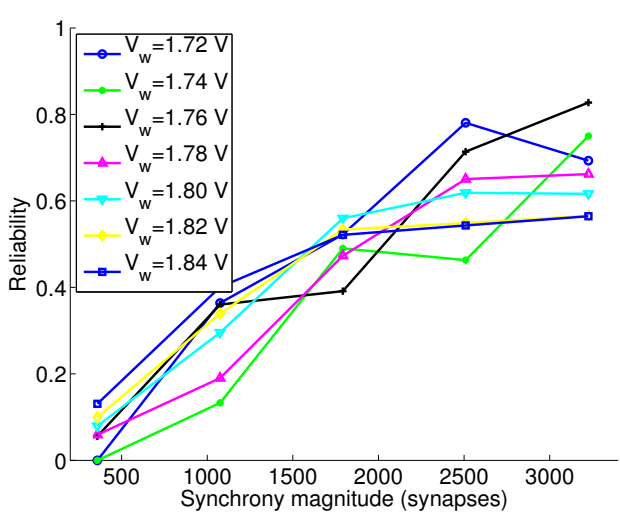

Fig. 3. Reliability as a function of synchrony magnitude for different synaptic weight parameter values.

rate-dependent increase in reliability can be observed with an increase in synchronous input. This indicates that spikes are aligning across trials in relative proportion to the spike rate to cause higher degrees of reliability.

\section{DISCUSSION}

The goal of this study was to characterize and juxtapose the roles of input spike synchrony and synaptic weights in generating precise and reliable spike timing in stereotyped cortical neurons which receive few direct signal inputs competing with noisy synaptic inputs from other parts of the brain. We found that reliability of the neuron spike patterns increased with an increase in synchronous inputs, regardless of how synaptic weights were varied. These findings are consistent with observed correlations measured for cortical cells [6] and suggest that synchrony can enhance the flow of information into the cortex from the thalamus even with weak synapses. These findings also fit with feed-forward connectivity models for simple cell orientation selectivity [16]. Synchrony also provides an explanation for the reliability and preservation of spike timing information observed in the primary visual pathway [4] as well as reliable feature selectivity. These synchronous events may propagate through the cortex in the form of a synfire chain [17] or be decoded by populations of cortical neurons that transform the sequence of input events into a new sequence of output events.

Through convergence and divergence, the LGN could create the degree of synchrony needed to drive V1 cells. This could be tested by studying the effects of modulating the intensity of visual stimuli on the firing rates and reliability of spike timing in cortical cells [18], [19]. Synchrony of cortical inputs is most likely to occur when there is an event of high importance in the sensory environment, such as the entrance of a moving object into a receptive field [20] or the deflection of a whisker in rodent [6]. Spiking due to input synchrony may be a way to insure that important events are registered by the cortical input neurons despite the disproportionately larger number of asynchronously arriving spikes from ongoing computation within the cortex. This prediction could be tested with intracellular recordings in vivo by using a dynamic 


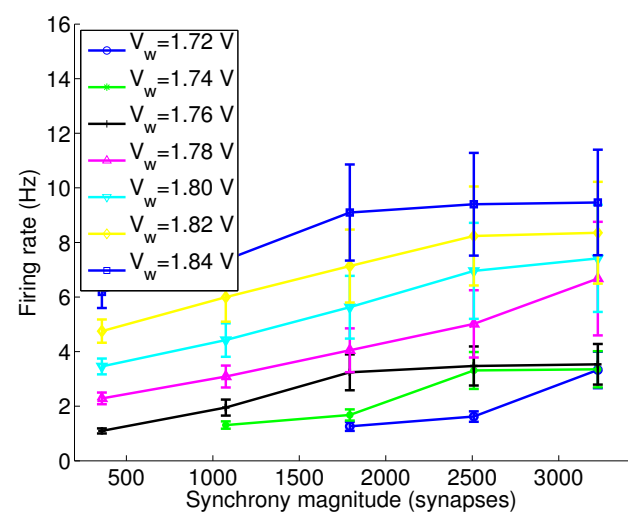

Fig. 4. Mean firing rates across trials versus synchrony magnitude, for increasing values of the synaptic weight parameter.

clamp to inject somatic conductances reflecting signal events in the presence of in vivo background noise. The reliability levels from these experiments can then be compared with those obtained from the model. The presence of realistic and constrained background synaptic activity components could validate the assumptions and parameters of the model and confirm the efficacy of synchrony in obtaining a high degree of reliability.

Spike synchrony, observed throughout the cortex, may regulate information transmission. For example, the coherence between the timing of spikes and synchronous oscillations in the field potentials in cortical area V4 increases with attention in monkeys [21]. Basket cells have strong inhibitory contacts on the somas of pyramidal cells and a small number of synchronously firing basket cells could strongly influence the spike timing in pyramidal cells located within a cortical column [22]. The impact of the synchronous spikes induced in the pyramidal cells could in turn have the same impact downstream as the synchronous LGN spikes have on the spiny stellate cells shown here.

Finally, we demonstrate that with further tuning and alignment with experimental evidence, a real-time neuromorphic system can be a useful platform for testing theories of information coding in biological systems. In fact, these systems may act as tools to test theories in ways not accessible by biological experiments. Synchrony, for example, is experimentally difficult to directly measure given the need to simultaneously record from a vast number of neurons and synapses. Furthermore, these findings could suggest different operating regimes for a spike based computational system and assist the implementation of embodied neuromorphic computing system that processes realistic inputs and provides output results within a natural environment.

\section{ACKNOWLEDGMENT}

The authors wish to thank Donald Spencer, and Jean-Marc Fellous for helpful discussions and project support. We would also like to acknowledge Telluride Neuromorphic Engineering Workshop, for giving the authors the opportunity to meet and start a fruitful collaboration. This work was supported by the
Howard Hughes Medical Institute NSF-IGERT grant, and by the EU DAISY grant FP6-2005-015803.

\section{REFERENCES}

[1] Z. F. Mainen and T. J. Sejnowski, "Reliability of spike timing in neocortical neurons," Science, vol. 268, no. 5216, pp. 1503-6, 1995.

[2] A. Peters and B. R. Payne, "Numerical relationships between geniculocortical afferents and pyramidal cell modules in cat primary visual cortex," Cereb. Cortex, vol. 3, no. 1, pp. 69-78, 1993.

[3] B. Ahmed, J. C. Anderson, R. J. Douglas, K. A. Martin, and J. C. Nelson, "Polyneuronal innervation of spiny stellate neurons in cat visual cortex," J. Comp. Neurol., vol. 341, no. 1, pp. 39-49, 1994

[4] P. Reinagel and R. C. Reid, "Precise firing events are conserved across neurons," Journal of Neuroscience, vol. 22, no. 16, pp. 6837-41, 2002.

[5] D. Ferster, S. Chung, and H. Wheat, "Orientation selectivity of thalamic input to simple cells of cat visual cortex," Nature, vol. 380 , no. 6571 , pp. 249-52, 1996.

[6] R. M. Bruno and B. Sakmann, "Cortex is driven by weak but synchronously active thalamocortical synapses," Science, vol. 312, no. 5780, pp. 1622-7, 2006.

[7] W. M. Usrey, J. B. Reppas, and R. C. Reid, "Paired-spike interactions and synaptic efficacy of retinal inputs to the thalamus," Nature, vol. 395, no. 6700, pp. 384-7, 1998

[8] J. M. Alonso, W. M. Usrey, and R. C. Reid, "Precisely correlated firing in cells of the lateral geniculate nucleus," Nature, vol. 383, no. 6603, pp. 815-9, 1996.

[9] G. Indiveri, E. Chicca, and R. Douglas, "A VLSI array of low-power spiking neurons and bistable synapses with spike-timing dependent plasticity," IEEE Transactions on Neural Networks, vol. 17, no. 1, pp. 211-221, Jan 2006.

[10] C. Bartolozzi and G. Indiveri, "Synaptic dynamics in analog VLSI," Neural Computation, vol. 19, pp. 2581-2603, Oct 2007.

[11] M. Mahowald, "VLSI analogs of neuronal visual processing: a synthesis of form and function," Ph.D. dissertation, Department of Computation and Neural Systems, California Institute of Technology, Pasadena, CA., 1992.

[12] A. Destexhe and D. Pare, "Impact of network activity on the integrative properties of neocortical pyramidal neurons in vivo," J Neurophysiol, vol. 81, no. 4, pp. 1531-47, 1999.

[13] S. Schreiber, D. Whitmer, J. M. Fellous, P. Tiesinga, and T. J. Sejnowski, "A new correlation-based measure of spike timing reliability." Neurocomputing, vol. 52-54, pp. 925-931, 2003.

[14] P. Reinagel, D. Godwin, S. M. Sherman, and C. Koch, "Encoding of visual information by lgn bursts," J Neurophysiol, vol. 81, no. 5, pp. 2558-69, 1999

[15] Z. Gil, B. W. Connors, and Y. Amitai, "Efficacy of thalamocortical and intracortical synaptic connections: quanta, innervation, and reliability," Neuron, vol. 23, no. 2, pp. 385-97, 1999.

[16] D. H. Hubel and T. N. Wiesel, "Receptive fields, binocular interaction and functional architecture in the cat's visual cortex," Journal of Physiology, vol. 160, pp. 106-54, 1962.

[17] Y. Aviel, C. Mehring, M. Abeles, and D. Horn, "On embedding synfire chains in a balanced network," Neural Comput, vol. 15, no. 6, pp. 132140, 2003.

[18] P. Kara, P. Reinagel, and R. C. Reid, "Low response variability in simultaneously recorded retinal, thalamic, and cortical neurons," Neuron, vol. 27 , no. 3 , pp. $635-46,2000$.

[19] E. Schneidman, M. J. n. Berry, R. Segev, and W. Bialek, "Weak pairwise correlations imply strongly correlated network states in a neural population," Nature, vol. 440, no. 7087, pp. 1007-12, 2006.

[20] P. Reinagel and R. C. Reid, "Temporal coding of visual information in the thalamus," J Neurosci, vol. 20, no. 14, pp. 5392-400, 2000.

[21] P. Fries, J. H. Reynolds, A. E. Rorie, and R. Desimone, "Modulation of oscillatory neuronal synchronization by selective visual attention," Science, vol. 291, no. 5508, pp. 1560-3, 2001.

[22] P. H. Tiesinga, J. M. Fellous, E. Salinas, J. V. Jose, and T. J. Sejnowski, "Inhibitory synchrony as a mechanism for attentional gain modulation," Journal of Physiology Paris, vol. 98, no. 4-6, pp. 296-314, 2004. 